\title{
Current Concepts in the Neuropathology and Pathogenesis of Multiple Sclerosis
}

\author{
G.R. Wayne Moore
}

\begin{abstract}
Multiple sclerosis (MS) has been classically regarded as an inflammatory demyelinating disease of the central nervous system. In recent years, the classification and pathogenesis of the disease have become controversial, particularly with respect to whether an individual patient demonstrates a single or multiple pathogenetic mechanisms in the establishment of the focal plaque of MS. It is also becoming increasingly apparent that there is a significant neurodegenerative component in the disease, involving not only plaques but the non-plaque parenchyma as well. Magnetic resonance imaging, together with histopathologic studies, will continue to shed light on the pathogenesis of these focal and diffuse abnormalities in MS.
\end{abstract}

RÉSUMÉ: Connaissances actuelles sur la neuropathologie et la pathogenèse de la sclérose en plaques. La sclérose en plaques (SP) est considérée comme une maladie démyélinisante inflammatoire du système nerveux central. Au cours des dernières années, la classification et la pathogenèse de la maladie ont fait l'objet de controverses, particulièrement en ce qui concerne la présence d'un ou de plusieurs mécanismes pathogènes dans la formation de la plaque focale de la SP. Il est de plus en plus évident qu'il existe une composante neurodégénérative significative dans cette maladie, impliquant non seulement les plaques mais aussi le parenchyme exempt de plaques. L'IRM a contribué et, conjointement avec les études anatomopathologiques, continuera à élucider la pathogenèse de ces anomalies focales et diffuses dans la SP.

Can. J. Neurol. Sci. 2010; 37: Suppl. 2 - S5-S15

It is indeed a pleasure to compile this review on the current concepts in the neuropathology and pathogenesis of multiple sclerosis (MS) in honour of Dr. Ken Warren, who has dedicated his professional life to the understanding of the biology of this disease and to the compassionate treatment of patients whom it afflicts. As a basic science researcher he has contributed to the understanding of the fascinating and complex biological perturbations in MS. As a clinician he has seen the true "human" toll of MS and has been a pioneer in the battle for its alleviation.

This article will be a brief overview of our current views on the neuropathology of MS and how these contribute to our understanding of its pathogenesis and its manifestations in modern imaging modalities. Needless to say, there are areas of controversy in this field which continue to fuel lively debate. These concepts will be pointed out and I will attempt to remain unbiased and leave it to the reader to come to his/her own conclusions based on the evidence at hand at present.

The following topics will be briefly covered:

1. The Classic Neuropathology of MS,

2. Neuropathology and Pathogenesis of MS: Current Concepts and Controversy,

3. Neuropathology of MS: Correlation with Magnetic Resonance Imaging (MRI),

4. Summary.

\section{The Classic Neuropathology of MS}

\section{A. Histopathology of MS}

The study of the pathology of MS dates back to the early 1800 's in the works of Carswell ${ }^{1}$ and Cruveilhier ${ }^{2}$ and was the subject of a beautifully illustrated monograph by Dawson in $1916^{3}$. Details of the history of MS pathology may be found in a recent review ${ }^{4}$.

The primary pathologic features of MS are chronic inflammation and demyelination accompanied by gliosis (Figure 1). The reader is referred to extensive reviews of the subject for more detailed information ${ }^{5-7}$. Briefly, the plaques of multiple sclerosis are macroscopically visible as focal lesions throughout

From the Department of Pathology and Laboratory Medicine, University of British Columbia; Division of Neuropathology, Vancouver General Hospital, Vancouver, British Columbia, Canada.

Received April 19, 2010. Final Revisions Submitted May 19, 2010. Correspondence to: G.R. Wayne Moore, Room 5450, Blusson Spinal Cord Centre, ICORD (International Collaboration on Repair Discoveries), 818 West 10th Avenue, Vancouver, British Columbia, V5Z 1M9, Canada. 
the central nervous system (CNS) white matter. The white matter most obviously involved is periventricular where the lesions, or plaques, are characterized by large areas of gray discolouration. However, anywhere in the CNS may be attacked.

Microscopically, plaques show axons that have undergone or are undergoing demyelination. The active demyelination is carried out by macrophages ${ }^{8}$ (Figure 1), which degrade the phagocytosed myelin to neutral lipid within their lysosomes ${ }^{9}$. It has been assumed that the macrophage is the effector cell of an autoimmune attack on the myelin-oligodendrocyte unit orchestrated by lymphocytes in the chronic inflammatory infiltrates in the perivascular spaces and parenchyma of the lesion (Figure 1F). Studies have shown that lymphocytic population of the MS lesion bear an array of markers corresponding to a variety of functional subtypes, including helper/inducer T-cells, with either $\alpha-\beta$ or $\sigma-\gamma$ T-cell receptor types , cytotoxic T-cells, natural-killer cells, B-cells and plasma cells $^{6}$, all of which either singly or in concert have been implicated in the immunopathogenesis of the lesion ${ }^{10}$. Accompanying all these changes is gliosis, initially in the form of large reactive astrocytes (Figure 1E) and eventually as fibrillary gliosis.

It is now widely recognized that remyelination occurs in the MS lesion and paradoxically this appears to be maximum during active ongoing demyelination and inflammation ${ }^{11,12}$. Remyelination is characterized by thin myelin sheaths and short internodes, which may also undergo subsequent demyelination ${ }^{13}$.

Still more recently has been the recognition that while the most obvious change in the MS lesion is demyelination, significant axonal loss also occurs (Figure 2), and this is, at least in part, due to the transection of axons from damage inflicted by the products of the inflammatory infiltrate ${ }^{14,15}$. This is, in turn, reflected in the finding of Wallerian degeneration in long tracts in the MS spinal cord.

\section{B. The Classification of MS Plaques}

The classification of MS white matter plaques has been largely based on the degree and location of the inflammatory and demyelinative activity, which are taken to reflect the age of the lesion. Thus, it has been customary to classify plaques as acute, chronic active and chronic silent (or "chronic inactive") ${ }^{6,16}$.

The term acute plaque has been reserved for the lesion of acute MS (sometimes referred to as "Marburg type" of $\mathrm{MS}^{17}$ ), which presents clinically as a single monophasic event with widespread lesions of more or less the same age. However, it may also refer to histopathologically similar lesions in the setting of chronic MS, these lesions presumably being the early stages of what will later be chronic MS plaques ${ }^{18}$. Acute MS lesions are relatively uniform in their histopathology throughout their extent, being regions of ongoing demyelination with intense hypercellularity attributable to prominent perivascular chronic inflammatory infiltrates, numerous macrophages with myelin debis and/or neutral lipid, monocytes, lymphocytes, reactive astrocytes, and increased numbers of oligodendroglia involved in remyelination (Figure 1A-F). Remyelination may be extensive and is responsible for "shadow plaques" 19 , which show relative reduction of stain intensity on myelin stains due to the presence of thin short internodes.

Correlating with the monophasic nature of acute MS, the lesions show a relative uniformity in histologic appearance in a given patient (Figure 1A-C). However, even in acute MS variability in lesion age, based on histologic features, may be evident, and in some cases these features may be consistent with a lesion developing before the time of emergence of clinical symptoms and signs ${ }^{20}$.

In contrast, the chronic active plaque shows a distinct topography reflecting the fact that it is seen in the chronic form of MS, where the lesion has time to mature. Thus, older histopathologic features are seen in the centre of the lesion, which shows varying number of lipid-laden macrophages and fibrillary gliosis. The border of the lesion shows ongoing demyelinative activity in the form of inflammation, with macrophages engaged in active myelin removal and degradation, reactive astrocytes, oligodendrocyte hyperplasia and remyelination - in many ways resembling the features seen throughout the acute lesion. This border of demyelinative activity extends centrifugally into the adjacent "normalappearing" white matter.

Figure 1: (opposite page) Illustrative classic neuropathology of MS. Acute MS: A-F: An acute MS plaque is characterized by a focal area of demyelination, demonstrated as loss of Luxol Fast Blue (LFB) staining (A), and perivascular cuffs of chronic inflammation (black arrows). The lesion has a uniform appearance throughout, containing numerous macrophages with LFB-positive myelin debris (red arrows), both in its centre (B) and at its edge $(C)$. At the lesion edge $(C)$, the macrophages (red arrows) are seen close to myelin sheaths in the adjacent white matter (yellow arrow). $D$, $E$ and $F$ show immunofluorescence in an acute plaque, with myelin basic protein (MBP in myelin) in green, heavy neurofilament (NF-H in axons) in red, combined with lavender for HAM 56 (macrophages) in D, or glial fibrillary acidic protein (astrocytes) in E, or CD45 (a pan-lymphocyte marker) in F. Note the macrophages containing MBP-positive debris amidst axons that have been demyelinated $(D)$, the prominent reactive astrocytes $(E)$, and the large number of perivascular lymphocytes $(F)$. Active MS lesion: G. Immunohistochemistry for Class II MHC shows an active lesion with positivity throughout $(G)$. Chronic active MS lesions: H, I and J: Class II MHC immunoreactivity is prominent at the border of several chronic active lesions (H). At the lesion edge there are Class II-positive activated microglia, which possess ramified processes, and macrophages which have a round appearance (I), whereas in the adjacent "normal-appearing white matter" only ramified microglia are positive for Class II MHC (J). Chronic silent MS plaque: K. The edge of a chronic silent lesion shows no evidence of inflammation, as evidenced by absence of hypercellularity $(K)$. In the lesion there is virtual absence of myelin in the lesion (upper half of $K$ ), the edge of which has a relatively abrupt border abutting the adjacent white matter (lower half of $K$ ) that shows axons (black) with myelin sheaths (blue). A, B, C: LFB-PAS; D, E, F: Triple immunofluorescence for MBP and NF-H, combined with HAM 56 (D), GFAP (E), or CD 45 (F); G, H, I, J: Immunohistochemistry for Class II MHC; K: LFB-Bielschowsky. Scale bars for high magnifications: Black $=400 \mu \mathrm{m}$, White $=25 \mu \mathrm{m}$. 


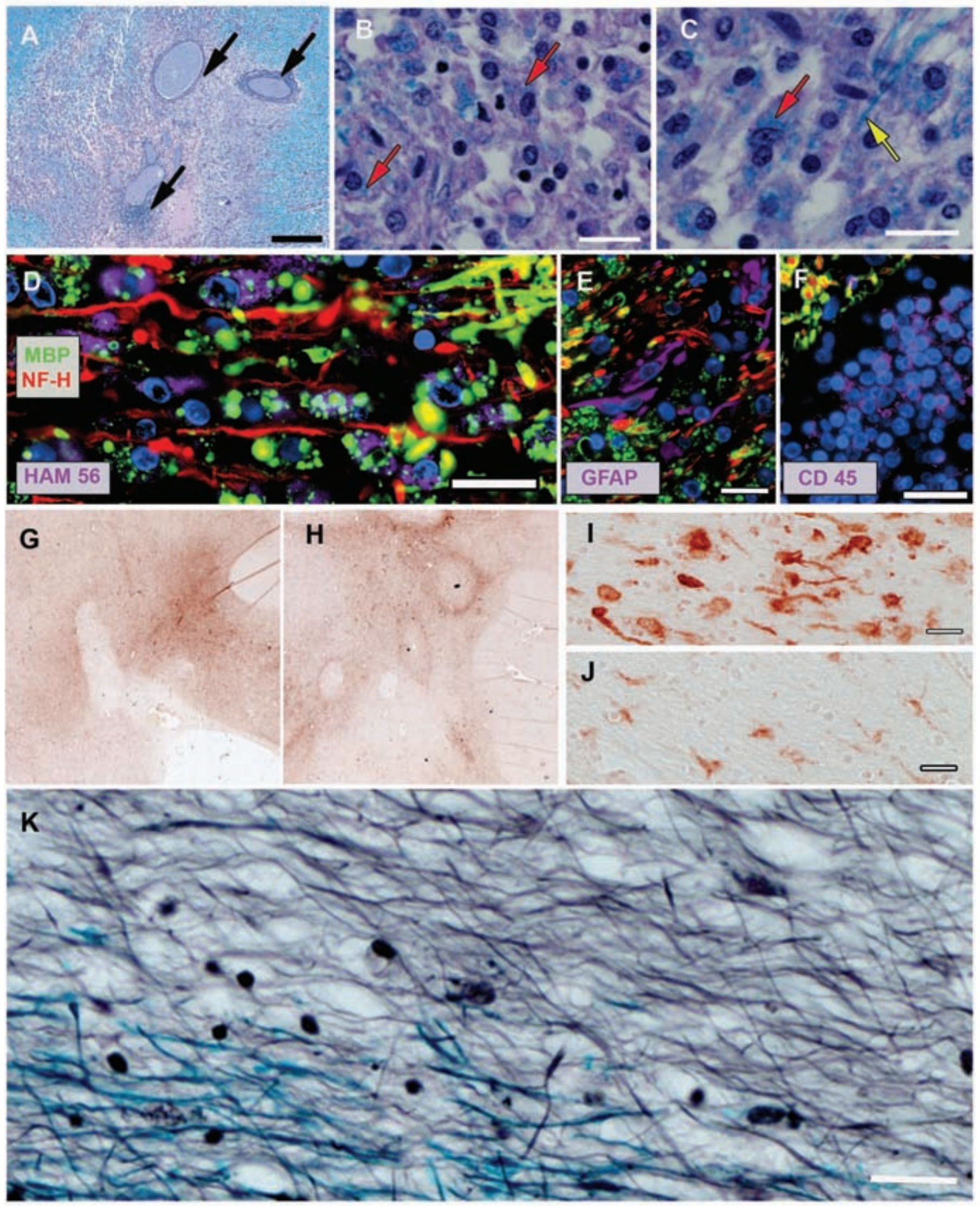


For reasons that are unclear, the immune-mediated demyelination at the lesion edge in chronic MS may evolve into a quiescent phase, at which point the term chronic silent plaque applies. Such lesions have very little inflammation, sometimes in the form of a few perivascular lymphocytes and lipid-laden macrophages, in an oligodendroglial-depleted expanse of demyelinated axons with fibrillary gliosis (Figure 1K). However, plasma cells, responsible for the continuous production of oligoclonal IgG, may be abundant in the chronic inactive lesion $^{21}$. The border of the lesion may show a variable degree of remyelination that had survived the demyelinatory onslaught during the active phase. If this is prominent, the plaque will have the appearance of a shadow plaque. It is important to note that the chronic inactive lesion may reactivate at any time with resumption of inflammatory demyelination, either focally or throughout its edge ${ }^{22}$.

A classification system based on the degree of expression of Class II major histocompatiblility complex (MHC) by microglia/macrophages and the degree of myelin breakdown to neutral lipid as shown by Oil red O-positivity was proposed in $1993^{23}$.

More recently, many MS researchers have employed a classification system that determines the age and activity of an MS plaque based on the reactivity of microglia and their maturation to macrophages as assessed by immunohistochemistry for the Class II $\mathrm{MHC}^{24}$. Here an active lesion is defined as a lesion with a uniform distribution of Class II MHCpositive microglia (Figure $1 \mathrm{G}$ ) indicating an early stage in the lesion evolution and corresponds to the acute plaque described above. A chronic active lesion has a border of intense Class II MHC positivity in macrophages and microglia (Figure H-J) and is the equivalent of the chronic active lesion with its edge of ongoing demyelination described above. A chronic inactive lesion is one in which Class II MHC expression is downregulated indicative of the dampening of the immune response in the lesion and corresponds to the chronic silent lesion.

\section{Neuropathology and Pathogenesis of MS:Current CONCEPTS AND CONTROVERSY}

In the last decade a classification system has been developed by researchers at the Mayo Clinic and University of Vienna ${ }^{25,26}$. This system, based on the examination of lesions undergoing active demyelination, defines four patterns of MS lesions. Patterns I and II have an immunopathogenetic basis, whereas Patterns III and IV have changes more indicative of a toxic of dystrophic effect on the oligodendroglial-myelin unit. Pattern I shows macrophage-mediated demyelination in the absence of antibody and complement deposition, whereas Pattern II is characterized by immunoglobulin and complement deposition, implicating a role for antibodies in its pathogenesis ${ }^{25}$. Both show an equivalent loss of the various myelin proteins ${ }^{26}$. The Pattern III lesion differs from the others in that it is not perivenous in location, has an ill-defined outline and shows a selective loss of myelin-associated glycoprotein $(\mathrm{MAG})^{26}$. There is extensive apoptosis of oligodendrocytes and oligodendrocyte loss in Pattern $\mathrm{III}^{26}$. This, together with the loss of MAG, which is located at the distal end of the oligodendrocyte process where it contacts the axon, suggests that this lesion results from a dyingback oligodendrogliopathy due to a perturbed oligodendroglial cell body ${ }^{26}$. The Pattern IV lesion, which is a very rare lesion, is characterized by non-apoptotic oligodendroglial cell death in the periplaque white matter and was only found in chronic progressive MS .

However, a point of considerable controversy arises from the concept that was also put forth by the Mayo-Vienna group when this system was formulated, which stated that MS was a heterogeneous disease among MS patients but was homogeneous within an individual MS patient ${ }^{25}$. In other words, the mechanism of myelin and oligodendrocyte destruction, as indicated by one of the four lesion types, was the same within all lesions of an individual MS patient.

A number of publications have supported this view. One was the finding that patients with Pattern II, who have deposits of immunoglobulins and complement in their lesions, improved clinically after therapeutic plasma exchange, a predictable result if these lesions had a humorally immune-mediated pathogenesis ${ }^{27}$. Patients with the other lesion patterns did not respond to this treatment ${ }^{27}$. There has also been a suggestion that MR imaging may be able to predict the pattern type ${ }^{28}$. Further, Pattern III lesions, but not Pattern II lesions, have been found to have mitochondrial defects, as evidenced by absent immunohistochemical staining for the catalytic subunit of the fourth complex of the mitochondrial respiratory chain ${ }^{29}$. This finding correlates with the notion that Pattern III lesions, which are also prominent in Balo's concentric sclerosis, may have a hypoxia-based pathogenesis ${ }^{30}$.

The concept of pathogenetic homogeneity within a given MS patient was challenged by an article by Barnett and Prineas in 2004, wherein they reported on the pathology of early lesions in relapsing-remitting MS. They observed oligodendrocyte apopotosis (a Pattern III feature) and complement deposition (a Pattern II feature) in the same patients ${ }^{31}$, a combination that was not evident in the Mayo-Vienna material ${ }^{32}$. The controversy of lesion heterogeneity aside, this article also raised the possibility that MS, in the first instance, may not be immunologically mediated, since they observed very few lymphocytes in the early lesions. However, they could not exclude a role for antibodies in the early pathogenetic events ${ }^{33,34}$. Exploring these possibilities further, this group very recently has shown that antibody and complement deposition are not specific for the MS lesion and is evident in disorders that are not thought to have an immunemediated pathogenesis, such as cerebral infarcts ${ }^{35}$, again questioning the validity of the Pattern II MS lesion and the role of antibodies in the pathogenesis of MS. Another group, based in Amsterdam, has studied a large group of lesions in established MS and has found that all chronic lesions show complement and antibodies in association with macrophages ${ }^{36}$. Therefore, they have concluded that in chronic MS all lesions are homogeneous in their pathogenesis ${ }^{36}$, essentially showing Pattern II histopathology. As noted in an accompanying editorial by Raine $^{37}$, this report represents a significant negative in the tally of pros and cons in the re-examination of the four-pattern patient-specific classification of MS lesions based on pathogenesis .

Thus, it is clear there is considerable disagreement at the moment about the heterogeneity/homogeneity of MS and even the importance of the immune system in the early pathogenesis of MS has come into question. There are a variety of reasons why 
this is so, and it may not necessarily be that one approach is absolutely "correct" and the other absolutely "wrong". Some of these disparities may find their explanation in the rarity of acute MS tissue (which is very often biopsy material), different immunohistochemical staining techniques, different antibodies and different antigens being examined, the vagaries of working with formalin-fixed tissue, the point in the evolution of the lesion in which it was sampled (early in studies with a predominance of biopsy material, or late in those with predominantly post-mortem material), and the variable factor of tissue autolysis in biopsy versus autopsy material ${ }^{38}$. However, it is probable that all these factors, either singly or in combination, do not account for all the discrepancies ${ }^{38}$.

Can these seemingly disparate findings and opinions be resolved? Is it possible that different research groups are seeing the same pathogenetic process at different time points is its maturation? Is it possible that during the immunopathogenesis of the disease or a single lesion different components of the immune system are operative in the progression from acute to chronic, and the different findings of various studies are simply a reflection of the sampling time ${ }^{38}$ ? Is it possible that MS in its early stages is not a disorder of the immune system but rather a neurodegenerative/toxic condition which then triggers the immune system into action? Clearly, much more work needs to be done to resolve the issues in this controversial, but fascinating, study of the early and subsequent phases in the pathogenesis of the MS plaque. As suggested by Esiri in a recent editorial, one approach to these controversies would be the formation of a panel, which could assess material from a variety of workers and institutions and produce a consensus, much as has been done in Alzheimer's Disease and other neurodegenerative disorders ${ }^{38}$.

\section{Neuropathology OF MS: CORRElation WITH MRI}

\section{A. The MS Plaque}

With the advent of MRI and its application to MS, it became clear that this technique was very sensitive in the detection of MS plaques (Figure 2). It was initially thought that specific abnormalities detected by MRI would have specific histologic correlates and that MRI would be a direct window on the pathology of MS. Early on post-mortem studies showed that MRI indeed detected MS plaques and the area and shape of these lesions on the MRI corresponded to that noted in the tissue ${ }^{39,40}$. Correlative studies showed that abnormalities seen on T2weighted images could be attributed variously to demyelination, macrophages, vascular permeability, edema, expansion of the extracellular space secondary to tissue loss or gliosis, and that there was no histologic feature which could produce a specific $\mathrm{T} 2$-weighted abnormality ${ }^{41}$. The same proved to be true for T1weighted imaging ${ }^{41}$, except for two notable exceptions. Firstly, permanent black holes on T1-weighted MRI corresponded to areas of severe parenchymal destruction with axonal $\operatorname{loss}^{42}$. Black holes which were transient were felt to have remyelination or edema resolution to account for their evanescence ${ }^{43}$. Secondly, contrast enhancement in T1-weighted MRI has been correlated with breakdown of blood-brain barrier secondary to inflammatory infiltrates ${ }^{44}$. Vascular neogenesis in the lesion also may contribute to contrast enhancement ${ }^{45}$.
However, specialized MRI techniques have been shown to have histological specificity or have the potential to do so. Intuitively, a technique which could detect a biochemical marker specific for a cell type should provide a high degree of specificity. One such technique is proton magnetic resonance spectroscopy $(\mathrm{MRS})^{46}$. A marker widely used to assess axonal loss by MRS is N-acetyl aspartate (NAA) ${ }^{47}$ and indeed this has been shown to be reduced in MS plaques by $\mathrm{MRS}^{48}$. Increased choline in MS plaques has been attributed to cell membranes in inflammatory infiltrates ${ }^{49}$ and elevated lactate to macrophages ${ }^{50}$. Choline has also been thought to be due to gliosis ${ }^{50}$, as has elevated myoinositol ${ }^{51}$ and creatine ${ }^{52}$. The abnormal lipid peaks on MRS are felt to reflect myelin breakdown ${ }^{49}$, and the reduced phospholipids in the plaque are evident on MRS for phosphorous $^{53}$.

Magnetization transfer imaging (MTI) detects the water associated with macromolecular structures. It has been correlated with the integrity of myelin in some studies ${ }^{54,55}$ and with axons in others ${ }^{56,57}$.

Diffusion-weighted imaging (DWI) measures the random motion of protons and abnormalities of its parameters, including apparent diffusion coefficient (ADC), mean diffusivity and fractional anisotropy (FA $)^{58}$, have been associated with myelin and to a lesser degree axonal pathology 59 .

A relaxation MRI technique has been developed that appears to be associated with the lamellar structure of myelin and seems to be specific for myelin ${ }^{60}$. Mathematically, it is possible to separate the T2 decay curve of the central nervous system into three components: a long (greater than $1 \mathrm{~ms}$ ) component from cerebrospinal fluid, and intermediate component (approximately $80-100 \mathrm{~ms}$ ) thought to originate in extracellular and intracellular fluid, and the myelin-specific short-T2 component (less than $50 \mathrm{~ms})^{60}$. The short T2 component has been shown qualitatively (Figure 2) (1 $^{61}$ and quantitatively ${ }^{62}$ to correlate with the presence of myelin in MRI-pathologic correlative studies and is absent ${ }^{61}$ in MS plaques with complete loss of myelin but surviving axons. Thus, the fraction of the T2 signal attributible to the short T2 component has also been referred to as the "myelin water fraction" (MWF). There is also evidence to indicate that the short $\mathrm{T} 2$ component recognizes remyelinated myelin, in as much as a serial study has shown its return in lesions which previously were devoid of this signal ${ }^{63}$.

Aside from histologic specificity, the MRI scan can detect patterns within MS lesions, which may correlate with the pathogenesis of a specific lesion. For example, a change in the MRI signal at the periphery of the lesion may indicate on-going demyelination ${ }^{64,65}$. The characteristic ring pattern of Balo's concentric sclerosis has also been confirmed by histopathologicMRI correlation ${ }^{66}$.

It has become increasingly apparent that in addition to classic white matter plaques of MS, demyelinative lesions are also present in the gray matter. These cortical plaques can be quite extensive and usually abut the pia, but occasionally may be evident deep to the superficial cortical layers ${ }^{67,68}$ (Figure 2). Plaques within subcortical U-fibres may also extend into the deeper cortical layers forming leucocortical plaques ${ }^{69}$. Cortical plaques are thought to be responsible for much of the cognitive dysfunction of MS as well as MS-associated epilepsy and thus contribute to a significant burden of the disease ${ }^{70,71}$. This is not 


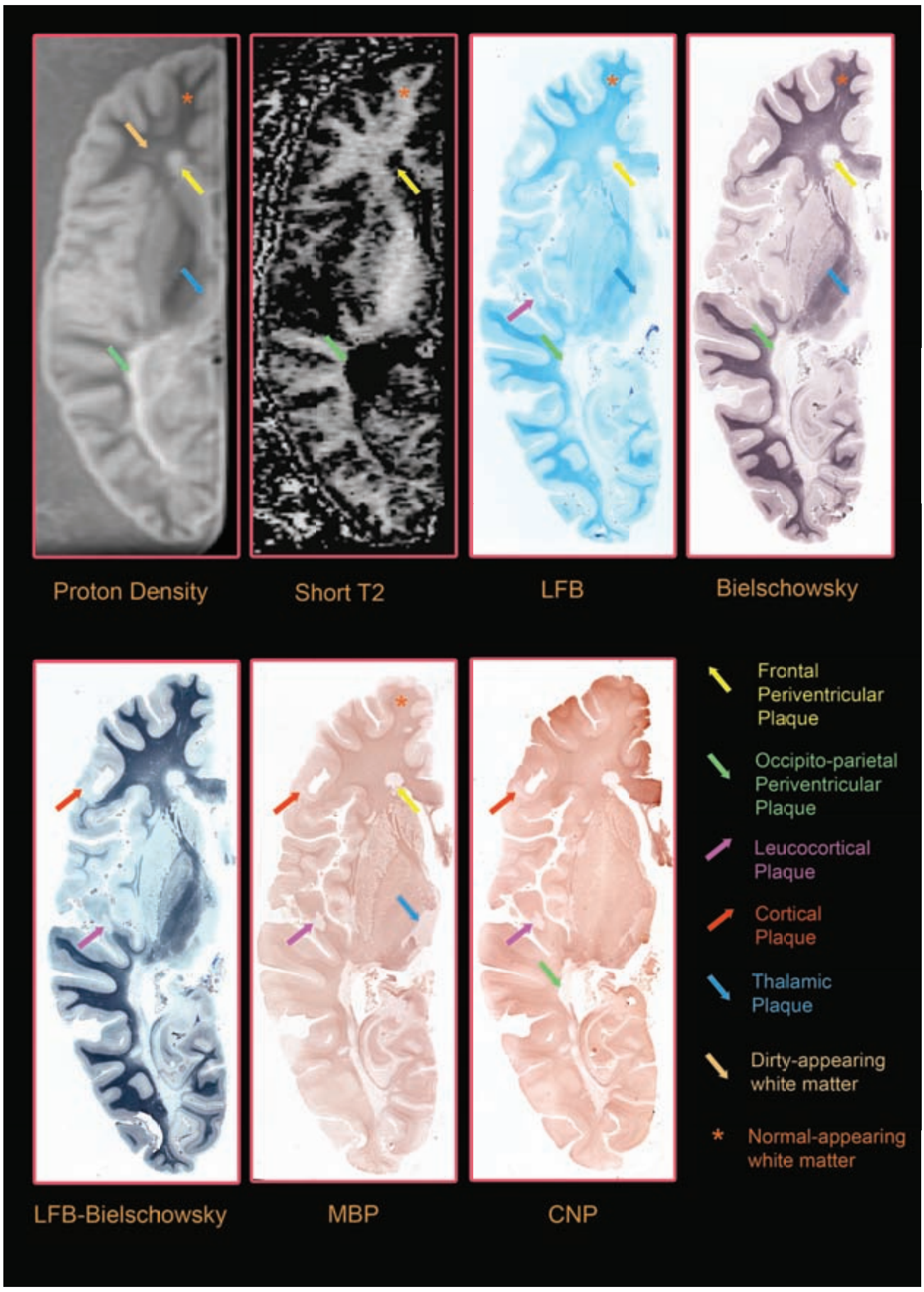


particularly surprising given the extensive neuronal and synaptic loss in these lesions ${ }^{72}$. They have also been particularly associated with the progressive forms of $\mathrm{MS}^{71}$. Histopathologically these are quite distinct from white matter plaques in that there is generally very little, if any, inflammation ${ }^{73}$ and are much more readily detected by immunohistochemical stains for myelin proteins than by the routine stain used for myelin, luxol fast blue $(\mathrm{LFB})^{74}$. The reason for the paucity of inflammation is unclear, but it has been proposed that early in their course that these are inflammatory but become inactive in the later stages of the disease when most cases are examined pathologically ${ }^{75}$.

Until recently, cortical plaques were largely invisible on $\mathrm{MRI}^{68}$. However, with high-field strength MRI, which is available at only a few centres at present, these can now be demonstated with amazing clarity and this has been confirmed by histopathologic correlation ${ }^{76}$.

Studies of MS plaques in the deep gray matter (Figure 2) have been few. To date the findings include microglial activation and macrophage-mediated demyelination ${ }^{77}$ and a degree of inflammation intermediate between the paucity of inflammatory infiltrates in cortical plaques and the extensive inflammation seen in white matter lesions ${ }^{78}$.

\section{B. The Non-plaque Parenchyma}

Of considerable interest over recent years are the abnormalities in non-lesional parenchyma in MS. Thus, the socalled "normal-appearing white matter" (NAWM) (Figure 2) shows abnormalities detected both on the MRI scan as well as histopathologically. While the recent interest in this area has been largely driven by MRI, histopathologic abnormalities in MS NAWM have been known for quite sometime, but have not been emphasized until recently. These abnormalities include gliosis, perivascular inflammation, perivascular lipofuscin deposition, some demyelination ${ }^{79,80}$, widespread mild inflammation $^{81}$, and microglial activation ${ }^{82,83}$. Lysosomes and lysosomal enzymes ${ }^{80,84-86}$, metalloproteinases ${ }^{87}$, breakdown of extracellular matrix proteins ${ }^{88}$ and blood-brain barrier breakdown ${ }^{89,90}$ have also been reported in NAWM. Of particular relevance is the finding of axonal loss in MS NAWM ${ }^{91}$, which is attributable to Wallerian degeneration in axons subsequent to their transection in plaques ${ }^{92}$. Consistent with this finding is the demonstration of reduced NAA in NAWM $^{93}$ by MRS, a abnormality which is often present very early in the disease course $^{94}$. These histopathologic and MRI findings in NAWM have highlighted the presence of a widespread neurodegenerative process that contributes significantly to the clinicopathologic spectrum of $\mathrm{MS}^{6}$. It is unclear at the present time whether this is secondary to or independent of the inflammatory demyelinating component that has been emphasized in the past. It is also possible both scenarios may be operative. Other abnormalities evident in NAWM by non-conventional MRI include abnormalities in lipids ${ }^{53,95}$, increased choline ${ }^{96}$, creatine $^{96}$ and myoinositol ${ }^{97}$ on MRS, a reduction in $\mathrm{MTR}^{98}$ on MTI, increased $\mathrm{ADC}^{99}$ and reduced $\mathrm{FA}^{100}$ on diffusion weighted imaging. Relaxations studies have shown prolongation of $\mathrm{T} 1^{101}$, reduction in the short $\mathrm{T} 2$ component $^{62}$ and an increase in the total water content ${ }^{62}$. At this point it is not known which histopathologic features are responsible for which MRI abnormalities. It would seem that axonal loss would be the basis for the NAA reduction. In any event, the abnormalities in NAWM are clinically relevant in MS, being correlated with disability $^{102}$ and cognitive dysfunction ${ }^{94}$.

There are also diffuse abnormalities in the "normal-appearing gray matter" (NAGM) evident on non-conventional MRI of the cortex in MS. These consist of reduction in NAA and DWI and MTI abnormalities ${ }^{94}$. It is unclear whether this a diffuse abnormality independent of numerous cortical plaques or whether there is an independent neurodegenerative process in the cortex. Moreover, it is unclear as to what histopathologic features are responsible for these diffuse cortical abnormalities.

Diffuse MRI abnormalities are also seen in the deep gray matter. In the thalamus there is thought to be an increase in iron content evident on routine clinical MRI, evident as T2hypointensity ${ }^{103}$. However, this has not been correlated with histopathology to date. Diffuse neuronal loss with atrophy and MRS abnormalities have also been documented in the thalamus in MS, indicative again of a neurodegenerative process ${ }^{104}$.

Whereas abnormalities in NAWM are detected only by nonconventional imaging, there are subtle abnormalities in the nonlesional white matter that are evident on routine proton density and T2-weighted imaging. This was termed "dirty-appearing white matter" when originally described by Zhao, Li and

Figure 2: (opposite page) Illustration of MRI-pathology correlation in MS in a formalin-fixed cerebral hemisphere. Note the periventricular plaque in the frontal white matter (yellow arrows) and the much larger periventricular plaque in the occipito-parietal white matter (green arrows), which are evident as increased signal intensity on the proton density scan and as absence of signal on the short T2 component distribution (myelin water fraction). Note the short T2 component distribution corresponds to the distribution of myelin. The white matter plaques show loss of myelin as evidenced by absence of staining on the luxol fast blue (LFB), myelin basic protein (MBP) and 2', 3'-cylic nucleotide 3' phosphohydrolase (CNP) stains. They also show severe axonal loss as shown by the absence of Bielschowsky staining. A plaque in the thalamus (blue arrow) shows similar imaging and staining characteristics. A cortical plaque (red arrow) best seen on the MBP and CNP stains is not detected by MR imaging. A leucocortical plaque (purple arrow) is more evident on the LFB stain than the cortical plaque, probably due to the demyelination of the myelin-rich subcortical $U$-fibres, but is also not evident on the MR scans. Dirty-appearing white matter (DAWM; gold arrow), located peripheral to the periventricular plaques and deep to the normal-appearing white matter (NAWM; orange asterisk), is characterized by a signal intensity intermediate in strength between that of plaque and NAWM. DAWM is seen as pallor on the LFB and the Bielschowsky stains. It is not well discriminated by the MBP and CNP stains, which show somewhat more intense staining in the subcortical U-fibres in regions where the DAWM approaches them. MR images courtesy of Dr.A.L.MacKay. Histological sections courtesy of Ms.E.Leung. Modified after Sobel RA, Moore GRW. Demyelinating Diseases . In Love S, Louis DN, Ellison DW, editors, Greenfield's Neuropathology. 8th Edition. London: Hodder Arnold; 2008. p 1513-1608 (6). Reproduced by permission of Edward Arnold (Publishers) Ltd. 
colleagues ${ }^{105}$, but more recently has been referred to as "diffusely-abnormal white matter" (DAWM). The abnormality is manifest as an increased signal intermediate between that of plaque and that of NAWM - essentially a signal intensity similar to gray matter, except located in white matter (Figure 2). DAWM is usually located around periventricular plaques, particularly posteriorly. Since DAWM is a relatively new concept, it is possible some prior MRI and histopathology studies may have included DAWM in NAWM in the assessment of non-plaque parenchyma. The pathology of DAWM shows myelin and axonal involvement ${ }^{106}$ with BBB breakdown ${ }^{107}$. When various stains for myelin are employed, DAWM shows a reduction in staining with LFB, which stains phospholipids ${ }^{108}$, but relative preservation of staining for $\mathrm{MBP}^{109}$, suggesting there is a selective lipid abnormality in DAWM myelin (Figure 2). Axonal staining may also be reduced in DAWM, but not as severe or widespread as the reduction of LFB staining (Figure 2), suggesting that axonal pathology follows or is secondary to the myelin lipid abnormality $^{109}$. Further, the DAWM abnormality is well demonstrated on the MWF map, particularly at high field strength $^{110}$, suggesting that the short $\mathrm{T} 2$ component may be recognizing a myelin lipid perturbation, which may be responsible for a disruption of the lamellar structure of myelin thought to be the origin of the short T2 component. Further work is ongoing in the characterization of DAWM and its relationship to the histopathology and the pathogenesis of MS.

\section{The Initial MS Lesion}

The initial lesion in MS is unknown. It is presumed this is the first event in the genesis of the plaque. The histopathology of such a lesion has yet to be revealed. It is probable that the early MRI changes in a focal MS plaque are represented by very early changes seen at the locale of what will later be an established MS plaque. One of the earliest such changes is a reduction in the MTR, which can occur months prior to enhancement on routine imaging ${ }^{111,112}$. Other very early findings include an increase in cerebral blood flow and volume ${ }^{113}$, DWI changes ${ }^{114}$ and an increase in choline on MRS ${ }^{115}$.

\section{A Proposed Schema for Disease Manifestation based MRI- Pathology Correlation}

Our current understanding of the evolution of disease in a given MS patient may be summarized as follows. Evidence would suggest that the very earliest focal changes of reduced MTR, DWI abnormalities and findings by MRS occur in a small region of what will later be evident as an area of focal enhancement. By the time enhancement has begun the lesion is probably what would be defined as an acute or active MS plaque, with uniform breakdown of the BBB associated with inflammation throughout the lesion. When the enhancement becomes localized to the lesion periphery the plaque has now entered the chronic active stage with ongoing inflammatory demyelination, BBB breakdown and neovascularization confined to the plaque edge. Myelin loss would be evident as reduction of the MWF and remyelination as regions with a return of the MWF. Reduction of MTR would be related to demyelination or axonal loss in the lesion. Demyelination would also be manifest as lipid peaks, whereas axonal loss as a reduction in NAA on MRS. Gliosis would cause increase in creatine, inositol and choline. Choline increase could also be due to inflammatory infiltrates. Expansion of the plaque into the adjacent parenchyma would be manifest as progressive increase in the diameter of the ring of enhancement. Lesion expansion may be also be evident as just increasing size on the T2-weighed image, as it is probable the low-grade ongoing BBB breakdown at the lesion edge may not be of a sufficient degree to be detected as enhancement in some lesions. Those lesions where there had been such extensive inflammation that there was parenchymal destruction would appear as permanent black holes on T1weighted images. The disappearance of a black hole may indicate remyelination or the resolution of edema. Some lesions are associated with DAWM, evident as intermediate intensity in the periplaque white matter on routine T2-weighed or proton density imaging, such areas showing a perturbation in myelin lipids with a lesser degree and extent of axonal loss. While all these changes are occurring in white matter lesions there are lesions forming in gray matter that are largely undetected by routine MRI but well visualized by high field strength magnets. Widespread changes in NAWM and NAGM are also relatively undetected by MRI currently employed in routine clinical settings.

\section{Summary}

In summary, MS is a disorder characterized by plaques of inflammatory demyelination with significant axonal degeneration that is present early in the disease. These plaques show features that indicate both the innate and adaptive immune responses are involved in its pathogenesis. It has been proposed that, while these mechanisms are heterogeneous in MS, the immunopathogenesis of plaques in a given patient is uniform. This concept has generated controversy and evidence for and against it has been discussed above. Recent histopathologic studies have provided data to indicate that MS plaques may not necessarily have an immunopathogenesis in the first instance and that the immune response in the disease may be a secondary phenomenon. Abnormalities in the non-plaque white matter are evident in DAWM and NAWM. In DAWM there appears to be lipid loss with a lesser degree of axonal loss. In NAWM there is significant axonal loss, which appears to be associated with clinical progression and is present early in the course of the disease. In addition to white matter abnormalities there is a significant burden of disease in the gray matter, both in the form of focal plaques and diffuse abnormalities.

In the future, histopathologic and MRI studies will further probe the pathogenesis of the focal and diffuse abnormalities in $\mathrm{MS}$, as to whether they are initially imunopathogenetic or neurodegenerative in nature and whether MS is a single disease or a final common pathway for a variety of etiologies and pathogenetic mechanisms.

\section{ACKNOWLEDGEMENTS}

The author thanks his colleagues in the the MRI Reseach Centre, the Department of Physics and Astronomy, and Medicine (Neurology) at the University of British Columbia, and the Multiple Sclerosis Clinic at UBC Hospital for fruitful collaborations, Esther Leung and Vlady Pavlova for technical 
assistance, the patients and their families who donated tissue for research, and the Multiple Sclerosis Society of Canada for research funding.

\section{REFERENCES}

1. Carswell R. Pathological anatomy. Ilustrations of the elementary forms of disease. London: Longman, Orme, Brown, Green and Longman; 1838.

2. Cruveilhier J. Anatomie pathologique du corps humain; descriptions avec figures lithographiees et coloriees; des diverses alterations morbides dont le corps humain est susceptible. Paris: JB Balliere; 1829-1842.

3. Dawson JW. The histology of multiple sclerosis. Trans R Soc Edin Reproduced by the Montreal Neurological Institute, Montreal, 1973. 1916;50:517-40 (with plates).

4. Compston A, Lassmann H, McDonald WI. The story of multiple sclerosis. In: Compston A, Confavreux C, Lassmann $\mathrm{H}$, et al, Editors. McAlpine's multiple sclerosis, 4th edition. Philadelphia: Churchill Livingstone Elsevier; 2006. p. 3-68.

5. Lassman H, Wekerle $\mathrm{H}$. The pathology of multiple sclerosis. In: Compston A, Confavreux C, Lassman H, et al, Editors. McAlpine's multiple sclerosis, 4th edition. Philadelphia: Churchill Livingstone Elsevier; 2006. p. 557-99.

6. Sobel RA, Moore GRW. Demyelinating diseases. In: Love S, Louis DN, Ellison DW, Editors. London: Hodder Arnold; 2008. p. 1513-608.

7. Ludwin SK, Raine CS. The neuropathology of multiple sclerosis. In: Raine CS, McFarland HF, Hohlfeld R, editors. Multiple Sclerosis A comprehensive text. Philadelphia: Saunders Elsevier; 2008. p. 151-7.

8. Prineas JW, Connell F. The fine structure of chronically active multiple sclerosis plaques. Neurology. 1978;28(9 (Part 2)): 68-75.

9. Lampert PW. Fine stucture of the demyelinating process. In: Hallpike JF, Adams CWM, Tourtellotte WW, editors. Multiple sclerosis: pathology, diagnosis and management. Baltimore: Williams and Wilkins; 1983. p. 29-46.

10. Frohman EM, Racke MK, Raine CS. Multiple sclerosis--the plaque and its pathogenesis. N Engl J Med. 2006 Mar 2;354(9):942-55.

11. Raine CS, Scheinberg L, Waltz JM. Multiple sclerosis. Oligodendrocyte survival and proliferation in an active established lesion. Lab Invest. 1981 Dec;45(6):534-46.

12. Franklin RJ, Ffrench-Constant C. Remyelination in the CNS: from biology to therapy. Nat Rev Neurosci. 2008 Nov;9(11):839-55.

13. Prineas JW, Kwon EE, Cho ES, et al. Continual breakdown and regeneration of myelin in progressive multiple sclerosis plaques. Ann N Y Acad Sci. 1984;436:11-32.

14. Ferguson B, Matyszak MK, Esiri MM, et al. Axonal damage in acute multiple sclerosis lesions. Brain. 1997 Mar;120 (Pt 3): 393-9.

15. Trapp BD, Peterson J, Ransohoff RM, et al. Axonal transection in the lesions of multiple sclerosis. N Engl J Med. 1998 Jan 29;338 (5):278-85.

16. Raine CS. Demyelinating Disease. In Textbook of Neuropathology. 3rd Edition. Baltimore: Williams \& Wilkins, pp 627-714. 3rd ed. Baltimore: Williams \& Wilkins 1997.

17. Marburg O. Die sogenannte "akute multiple sklerose" (Encephalomyelitis periaxialis scleroticans). Jahrbucher fur Psychiatrie und Neurologie. 1906;27:213-312.

18. Moore GRW. Neuropathology and pathophysiology of the multiple sclerosis lesion. In: Paty DW, Ebers GC, editors. Multiple sclerosis. Philadelphia: F.A. Davis; 1998. p. 257-327.

19. Prineas JW. The neuropathology of multiple sclerosis. In: Koetsier JC, editor. Handbook of clinical neurology, Volume 3 (47). Amsterdam: Elsevier; 1985. p. 213-57.

20. Prineas JW. Pathology of multiple sclerosis. In: Cook SD, editor. Handbook of multiple sclerosis. New York: Marcel Decker; 1990. p. 187-218.

21. Prineas JW, Wright RG. Macrophages, lymphocytes and plasma cells in the perivascular compartment in chronic multiple sclerosis. Lab Invest. 1978(38):409-21.
22. Prineas JW, Barnard RO, Revesz T, et al. Multiple sclerosis. Pathology of recurrent lesions. Brain. 1993 Jun;116 (Pt 3): 681-93.

23. Sanders V, Conrad AJ, Tourtellotte WW. On classification of postmortem multiple sclerosis plaques for neuroscientists. J Neuroimmunol. 1993 Jul;46(1-2):207-16.

24. Bo L, Mork S, Kong PA, et al. Detection of MHC class II-antigens on macrophages and microglia, but not on astrocytes and endothelia in active multiple sclerosis lesions. J Neuroimmunol. 1994 May;51(2):135-46.

25. Lucchinetti C, Bruck W, Parisi J, et al. Heterogeneity of multiple sclerosis lesions: implications for the pathogenesis of demyelination. Ann Neurol. 2000 Jun;47(6):707-17.

26. Lucchinetti CF, Parisi J, Bruck W. The pathology of multiple sclerosis. Neurol Clin. 2005 Feb;23(1):77-105, vi.

27. Keegan M, Konig F, McClelland R, et al. Relation between humoral pathological changes in multiple sclerosis and response to therapeutic plasma exchange. Lancet. 2005 Aug 13-19;366 (9485):579-82.

28. Bruck W, Neubert K, Berger $\mathrm{T}$, et al. Clinical, radiological, immunological and pathological findings in inflammatory CNS demyelination--possible markers for an antibody-mediated process. Mult Scler. 2001 Jun;7(3):173-7.

29. Mahad D, Ziabreva I, Lassmann H, et al. Mitochondrial defects in acute multiple sclerosis lesions. Brain. 2008 Jul;131(Pt 7): 1722-35.

30. Stadelmann C, Ludwin S, Tabira T, et al. Tissue preconditioning may explain concentric lesions in Balo's type of multiple sclerosis. Brain. 2005 May;128(Pt 5):979-87.

31. Barnett MH, Prineas JW. Relapsing and remitting multiple sclerosis: pathology of the newly forming lesion. Ann Neurol. 2004 Apr;55(4):458-68.

32. Lucchinetti CF, Bruck W, Lassmann H. Evidence for pathogenic heterogeneity in multiple sclerosis (letter). Ann Neurol. 2004;56 (2):308.

33. Pender MP. Oliogodendrocyte apopotosis before immune attack in multiple sclerosis? (letter). Ann Neurol 2005;57(1):158.

34. Prineas JW, Barnett MH. Reply (letter). Ann Neurol. 2005;57 (158-159).

35. Barnett MH, Parratt JD, Cho ES, et al. Immunoglobulins and complement in postmortem multiple sclerosis tissue. Ann Neurol. 2009 Jan;65(1):32-46.

36. Breij EC, Brink BP, Veerhuis R, et al. Homogeneity of active demyelinating lesions in established multiple sclerosis. Ann Neurol. 2008 Jan;63(1):16-25.

37. Raine CS. Multiple sclerosis: classification revisited reveals homogeneity and recapitulation. Ann Neurol. 2008 Jan;63(1): $1-3$.

38. Esiri MM. MS: is it one disease? Int MS J. 2009 Jun;16(2):39-41.

39. Stewart WA, Hall LD, Berry K, et al. Correlation between NMR scan and brain slice data in multiple sclerosis. Lancet. 1984 (ii): 412 .

40. Paty DW, Moore GRW. Magnetic resonance imaging changes as living pathology in multiple sclerosis In: Paty DW, Ebers GC, editors. Multiple Sclerosis. Philadelphia: F.A. Davis; 1998. p. $328-69$.

41. Moore GR. MRI-clinical correlations: more than inflammation alone-what can MRI contribute to improve the understanding of pathological processes in MS? J Neurol Sci. 2003 Feb 15;206(2): $175-9$.

42. van Walderveen MA, Kamphorst W, Scheltens P, et al. Histopathologic correlate of hypointense lesions on T1-weighted spin-echo MRI in multiple sclerosis. Neurology. 1998 May;50 (5): $1282-8$

43. Bitsch A, Kuhlmann T, Stadelmann C, et al. A longitudinal MRI study of histopathologically defined hypointense multiple sclerosis lesions. Ann Neurol. 2001 Jun;49(6):793-6.

44. Katz D, Taubenberger JK, Cannella B, et al. Correlation between magnetic resonance imaging findings and lesion development in chronic, active multiple sclerosis. Ann Neurol. 1993 Nov;34(5): 661-9. 
45. Ludwin SK, Henry JM, McFarland HF. Vascular proliferation and angiogenesis in MS: clinical and pathogenetic implications (abstract). J Neuropathol Exp Neurol. 2001;60:505.

46. Sajja BR, Wolinsky JS, Narayana PA. Proton magnetic resonance spectroscopy in multiple sclerosis. Neuroimaging Clin N Am. $2009 \mathrm{Feb} ; 19(1): 45-58$

47. Bjartmar C, Battistuta J, Terada N, et al. N-acetylaspartate is an axon-specific marker of mature white matter in vivo: a biochemical and immunohistochemical study on the rat optic nerve. Ann Neurol. 2002 Jan;51(1):51-8.

48. Arnold DL, Matthews PM, Francis GS, et al. Proton magnetic resonance spectroscopic imaging for metabolic characterization of demyelinating plaques. Ann Neurol. 1992 Mar;31(3):235-41.

49. Narayana PA, Doyle TJ, Lai D, et al. Serial proton magnetic resonance spectroscopic imaging, contrast-enhanced magnetic resonance imaging, and quantitative lesion volumetry in multiple sclerosis. Ann Neurol. 1998 Jan;43(1):56-71.

50. Bitsch A, Bruhn $\mathrm{H}$, Vougioukas $\mathrm{V}$, et al. Inflammatory CNS demyelination: histopathologic correlation with in vivo quantitative proton MR spectroscopy. AJNR Am J Neuroradiol. 1999 Oct;20(9):1619-27.

51. Brex PA, Parker GJ, Leary SM, et al. Lesion heterogeneity in multiple sclerosis: a study of the relations between appearances on T1 weighted images, T1 relaxation times, and metabolite concentrations. J Neurol Neurosurg Psychiatry. 2000 May;68(5): 627-32.

52. Helms G, Stawiarz L, Kivisakk P, et al. Regression analysis of metabolite concentrations estimated from localized proton MR spectra of active and chronic multiple sclerosis lesions. Magn Reson Med. 2000 Jan;43(1):102-10.

53. Husted CA, Goodin DS, Hugg JW, et al. Biochemical alterations in multiple sclerosis lesions and normal-appearing white matter detected by in vivo $31 \mathrm{P}$ and $1 \mathrm{H}$ spectroscopic imaging. Ann Neurol. 1994 Aug;36(2):157-65.

54. Schmierer K, Scaravilli F, Altmann DR, et al. Magnetization transfer ratio and myelin in postmortem multiple sclerosis brain. Ann Neurol. 2004 Sep;56(3):407-15

55. Schmierer K, Wheeler-Kingshott CA, Tozer DJ, et al. Quantitative magnetic resonance of postmortem multiple sclerosis brain before and after fixation. Magn Reson Med. 2008 Feb;59(2): 268-77.

56. van Waesberghe JH, Kamphorst W, De Groot CJ, et al. Axonal loss in multiple sclerosis lesions: magnetic resonance imaging insights into substrates of disability. Ann Neurol. 1999 Nov;46 (5):747-54.

57. Fisher E, Chang A, Fox RJ, et al. Imaging correlates of axonal swelling in chronic multiple sclerosis brains. Ann Neurol. 2007 Sep;62(3):219-28.

58. Rovaris M, Gass A, Bammer R, et al. Diffusion MRI in multiple sclerosis. Neurology. 2005 Nov 22;65(10):1526-32.

59. Schmierer K, Wheeler-Kingshott CA, Boulby PA, et al. Diffusion tensor imaging of post mortem multiple sclerosis brain. Neuroimage. 2007 Apr 1;35(2):467-77.

60. MacKay A, Whittall K, Adler J, et al. In vivo visualization of myelin water in brain by magnetic resonance. Magn Reson Med. 1994 Jun;31(6):673-7.

61. Moore GR, Leung E, MacKay AL, et al. A pathology-MRI study of the short-T2 component in formalin-fixed multiple sclerosis brain. Neurology. 2000 Nov 28;55(10):1506-10.

62. Laule C, Leung E, Lis DK, et al. Myelin water imaging in multiple sclerosis: quantitative correlations with histopathology. Mult Scler. 2006 Dec;12(6):747-53.

63. Vavasour IM, Laule C, Li DK, et al. Longitudinal changes in myelin water fraction in two MS patients with active disease. J Neurol Sci. 2009 Jan 15;276(1-2):49-53.

64. Nesbit GM, Forbes GS, Scheithauer BW, et al. Multiple sclerosis: histopathologic and MR and/or CT correlation in 37 cases at biopsy and three cases at autopsy. Radiology. 1991 Aug;180(2): 467-74.

65. Bruck W, Bitsch A, Kolenda H, et al. Inflammatory central nervous system demyelination: correlation of magnetic resonance imaging findings with lesion pathology. Ann Neurol. 1997 Nov; 42(5):783-93.
66. Karaarslan E, Altintas A, Senol U, et al. Balo's concentric sclerosis: clinical and radiologic features of five cases. AJNR Am J Neuroradiol. 2001 Aug;22(7):1362-7.

67. Kidd D, Barkhof F, McConnell R, et al. Cortical lesions in multiple sclerosis. Brain. 1999 Jan;122 (Pt 1):17-26.

68. Geurts JJ, Bo L, Pouwels PJ, et al. Cortical lesions in multiple sclerosis: combined postmortem MR imaging and histopathology. AJNR Am J Neuroradiol. 2005 Mar;26(3):572-7.

69. Peterson JW, Bo L, Mork S, et al. Transected neurites, apoptotic neurons, and reduced inflammation in cortical multiple sclerosis lesions. Ann Neurol. 2001 Sep;50(3):389-400.

70. Roosendaal SD, Moraal B, Pouwels PJ, et al. Accumulation of cortical lesions in MS: relation with cognitive impairment. Mult Scler. 2009 Jun;15(6):708-14

71. Kutzelnigg A, Lassmann H. Cortical demyelination in multiple sclerosis: a substrate for cognitive deficits? J Neurol Sci. 2006 Jun 15;245(1-2):123-6.

72. Wegner C, Esiri MM, Chance SA, et al. Neocortical neuronal, synaptic, and glial loss in multiple sclerosis. Neurology. 2006 Sep 26;67(6):960-7.

73. Bo L, Vedeler CA, Nyland H, et al. Intracortical multiple sclerosis lesions are not associated with increased lymphocyte infiltration. Mult Scler. 2003 Aug;9(4):323-31.

74. Bo L, Vedeler CA, Nyland HI, et al. Subpial demyelination in the cerebral cortex of multiple sclerosis patients. J Neuropathol Exp Neurol. 2003 Jul;62(7):723-32.

75. Roemer S, Stadelmann C, Bruck W, et al. Cortical demyelination is present in early multiple sclerosis (abstract). Neurology. 2006 March;66 (Suppl 2)(5):A93-4.

76. Kangarlu A, Bourekas EC, Ray-Chaudhury A, et al. Cerebral cortical lesions in multiple sclerosis detected by MR imaging at 8 Tesla. AJNR Am J Neuroradiol. 2007 Feb;28(2):262-6.

77. Huitinga I, De Groot CJ, Van der Valk P, et al. Hypothalamic lesions in multiple sclerosis. J Neuropathol Exp Neurol. 2001 Dec;60 (12): 1208-18

78. Vercellino M, Masera S, Lorenzatti $M$, et al. Demyelination, inflammation, and neurodegeneration in multiple sclerosis deep gray matter. J Neuropathol Exp Neurol. 2009 May;68(5): 489-502.

79. Allen IV, Glover G, Anderson R. Abnormalities in the macroscopically normal white matter in cases of mild or spinal multiple sclerosis (MS). Acta Neuropathol Suppl (Berl). 1981;7: 176-8.

80. Allen IV, McKeown SR. A histological, histochemical and biochemical study of the macroscopically normal white matter in multiple sclerosis. J Neurol Sci. 1979 Mar;41(1):81-91.

81. Kutzelnigg A, Lucchinetti CF, Stadelmann C, et al. Cortical demyelination and diffuse white matter injury in multiple sclerosis. Brain. 2005 Nov; 128(Pt 11):2705-12.

82. Allen IV, McQuaid S, Mirakhur M, et al. Pathological abnormalities in the normal-appearing white matter in multiple sclerosis. Neurol Sci. 2001 Apr;22(2):141-4.

83. Gobin SJ, Montagne L, Van Zutphen M, et al. Upregulation of transcription factors controlling MHC expression in multiple sclerosis lesions. Glia. 2001 Oct;36(1):68-77.

84. Arstila AU, Riekkinen P, Rinne UK, et al. Studies on the pathogenesis of multiple sclerosis. Participation of lysosomes on demyelination in the central nervous system white matter outside plaques. Eur Neurol. 1973;9(1):1-20.

85. Riekkinen PJ, Rinne UK, Arstila AU. Neurochemical and morphological studies on demyelination in multiple sclerosis with special reference to etiological aspects. Z Neurol. 1972;203 (2):91-104

86. Rinne UK, Riekkinen PJ, Arstila AU. Biochemical and electron microscopic alterations in the white matter outside demyelinated plaques in multiple sclerosis. . In: Lubowitz U, editor. Progress in Multiple Sclerosis. New York: Academic Press; 1972. p. 76-98.

87. Lindberg RL, De Groot CJ, Montagne L, et al. The expression profile of matrix metalloproteinases (MMPs) and their inhibitors (TIMPs) in lesions and normal appearing white matter of multiple sclerosis. Brain. 2001 Sep;124(Pt 9):1743-53. 
88. Sobel RA, Ahmed AS. White matter extracellular matrix chondroitin sulfate/dermatan sulfate proteoglycans in multiple sclerosis. J Neuropathol Exp Neurol. 2001 Dec;60(12): 1198-207.

89. Plumb J, McQuaid S, Mirakhur M, Kirk J. Abnormal endothelial tight junctions in active lesions and normal-appearing white matter in multiple sclerosis. Brain Pathol. 2002 Apr;12(2): 154-69.

90. Kirk J, Plumb J, Mirakhur M, et al. Tight junctional abnormality in multiple sclerosis white matter affects all calibres of vessel and is associated with blood-brain barrier leakage and active demyelination. J Pathol. 2003 Oct;201(2):319-27.

91. Evangelou N, Esiri MM, Smith S, et al. Quantitative pathological evidence for axonal loss in normal appearing white matter in multiple sclerosis. Ann Neurol. 2000 Mar;47(3):391-5.

92. Evangelou N, Konz D, Esiri MM, et al. Regional axonal loss in the corpus callosum correlates with cerebral white matter lesion volume and distribution in multiple sclerosis. Brain. 2000 Sep; 123 (Pt 9): 1845-9.

93. De Stefano N, Narayanan S, Francis SJ, et al. Diffuse axonal and tissue injury in patients with multiple sclerosis with low cerebral lesion load and no disability. Arch Neurol. 2002 Oct;59(10): 1565-71.

94. Miller DH, Thompson AJ, Filippi M. Magnetic resonance studies of abnormalities in the normal appearing white matter and grey matter in multiple sclerosis. J Neurol. 2003 Dec;250(12): 1407-19.

95. Narayana PA, Wolinsky JS, Rao SB, et al. Multicentre proton magnetic resonance spectroscopy imaging of primary progressive multiple sclerosis. Mult Scler. 2004 Jun;10 Suppl $1:$ S73-8.

96. Inglese M, Li BS, Rusinek H, et al. Diffusely elevated cerebral choline and creatine in relapsing-remitting multiple sclerosis. Magn Reson Med. 2003 Jul;50(1):190-5.

97. Fernando KT, McLean MA, Chard DT, et al. Elevated white matter myo-inositol in clinically isolated syndromes suggestive of multiple sclerosis. Brain. 2004 Jun;127(Pt 6):1361-9.

98. Ropele S, Fazekas F. Magnetization transfer MR imaging in multiple sclerosis. Neuroimaging Clin N Am. 2009 Feb;19(1): 27-36.

99. Werring DJ, Clark CA, Droogan AG, et al. Water diffusion is elevated in widespread regions of normal-appearing white matter in multiple sclerosis and correlates with diffusion in focal lesions. Mult Scler. 2001 Apr;7(2):83-9.

100. Guo AC, Jewells VL, Provenzale JM. Analysis of normal-appearing white matter in multiple sclerosis: comparison of diffusion tensor MR imaging and magnetization transfer imaging. AJNR Am J Neuroradiol. 2001 Nov-Dec;22(10):1893-900.

101.van Walderveen MA, van Schijndel RA, Pouwels PJ, et al. Multislice T1 relaxation time measurements in the brain using IR-EPI: reproducibility, normal values, and histogram analysis in patients with multiple sclerosis. J Magn Reson Imaging. 2003 Dec;18(6):656-64.
102. Traboulsee A, Dehmeshki J, Peters KR, et al. Disability in multiple sclerosis is related to normal appearing brain tissue MTR histogram abnormalities. Mult Scler. 2003 Dec;9(6):566-73.

103. Bakshi R, Benedict RH, Bermel RA, et al. T2 hypointensity in the deep gray matter of patients with multiple sclerosis: a quantitative magnetic resonance imaging study. Arch Neurol. 2002 Jan;59(1):62-8

104. Cifelli A, Arridge M, Jezzard P, et al. Thalamic neurodegeneration in multiple sclerosis. Ann Neurol. 2002 Nov;52(5):650-3.

105. Zhao GJ, Li DKB, Cheng Y, et al. MRI dirty-appearing white matter in MS (abstract). Neurology. 2000;54 (suppl 3):A121.

106. Seewann A, Vrenken H, van der Valk P, et al. Diffusely abnormal white matter in chronic multiple sclerosis: imaging and histopathologic analysis. Arch Neurol. 2009 May;66(5):601-9.

107. Vos CM, Geurts JJ, Montagne L, et al. Blood-brain barrier alterations in both focal and diffuse abnormalities on postmortem MRI in multiple sclerosis. Neurobiol Dis. 2005 Dec; 20(3):953-60.

108. Segarra JM. Histological and histochemical staining methods: A selection In: Teduchi CG, editor. Neuropathology Methods and Diagnosis. Boston: Little, Brown and Company; 1970. p. 233-69.

109. Moore GR, Laule C, Mackay A, et al. Dirty-appearing white matter in multiple sclerosis: preliminary observations of myelin phospholipid and axonal loss. J Neurol. 2008 Nov;255(11):180211, discussion 12 .

110. Laule C, Kozlowski P, Leung E, et al. Myelin water imaging of multiple sclerosis at $7 \mathrm{~T}$ : correlations with histopathology. Neuroimage. 2008 May 1;40(4):1575-80.

111. Filippi M, Rocca MA, Martino G, et al. Magnetization transfer changes in the normal appearing white matter precede the appearance of enhancing lesions in patients with multiple sclerosis. Ann Neurol. 1998 Jun;43(6):809-14.

112. Laule C, Vavasour IM, Whittall KP, et al. Evolution of focal and diffuse magnetisation transfer abnormalities in multiple sclerosis. J Neurol. 2003 Aug;250(8):924-31.

113. Wuerfel J, Bellmann-Strobl J, Brunecker P, et al. Changes in cerebral perfusion precede plaque formation in multiple sclerosis: a longitudinal perfusion MRI study. Brain. 2004 Jan; 127(Pt 1):111-9.

114. Werring DJ, Brassat D, Droogan AG, et al. The pathogenesis of lesions and normal-appearing white matter changes in multiple sclerosis: a serial diffusion MRI study. Brain. 2000 Aug;123 (Pt 8):1667-76

115. Tartaglia MC, Narayanan S, De Stefano N, et al. Choline is increased in pre-lesional normal appearing white matter in multiple sclerosis. J Neurol. 2002 Oct;249(10):1382-90. 